\title{
Fast Motion Vector Estimation Using Multiresolution-Spatio-Temporal Correlations
}

\author{
Junavit Chalidabhongse, Member, IEEE, and C.-C. Jay Kuo, Senior Member, IEEE
}

\begin{abstract}
In this paper, we propose a new fast algorithm for block motion vector (MV) estimation based on the correlations of the MV's existing in spatially and temporally adjacent as well as hierarchically related blocks. We first establish a basic framework by introducing new algorithms based on spatial correlation and then spatio-temporal correlations before integrating them with a multiresolution scheme for the ultimate algorithm. The main idea is to effectively exploit the information obtained from the corresponding block at a coarser resolution level and spatiotemporal neighboring blocks at the same level in order to select a good set of initial MV candidates and then perform further local search to refine the MV result. We show with experimental results that, in comparison with the full search algorithm, the proposed algorithm achieves a speed-up factor ranging from 150 to 310 with only 2-7\% mean square error (MSE) increase and a similar rate-distortion performance when applied to typical test video sequences.
\end{abstract}

Index Terms - Block matching, motion estimation, multiresolution, spatial correlation, temporal correlation, video coding.

\section{INTRODUCTION}

$\mathbf{V}$ IDEO image compression plays an important role in transmission and storage of digital video data. The applications include multimedia transmission, teleconferencing, videophone, high-definition television (HDTV), CD-ROM storages, etc. The main idea to achieve compression is to remove temporal and spatial redundancies existing in video sequences. One effective method commonly used in reducing temporal redundancy is motion-compensated predictive coding, which is also employed in the MPEG standard [3], [10], [11]. The key ingredient in motioncompensated coding is motion vector (MV) estimation. The block matching technique has been widely used for MV estimation due to its simplicity. A straightforward way to obtain MV is to perform the full-search block matching algorithm (FBMA) by searching all locations in a given search area and selecting the position where the matching residual error is minimized. However, this procedure requires an extremely large amount of computation. MV estimation is known to be the main bottleneck in real-time encoding

Manuscript received October 16, 1995; revised April 26, 1996. This paper was recommended by Associate Editor B. G. Haskell. This work was supported by Rockwell, the Integrated Media Systems Center, a National Science Foundation Engineering Research Center, and the National Science Foundation Presidential Faculty Fellow Award ASC-9350309.

The authors are with the Integrated Media Systems Center and the Department of Electrical Engineering-Systems, University of Southern California, Los Angeles, CA 90089-2564 USA.

Publisher Item Identifier S 1051-8215(97)02552-4. applications, and the search for an effective MV estimation algorithm has been a challenging problem for years.

Fast block matching algorithms have been developed [12] to reduce the computational cost. They can be categorized into different groups as detailed below.

\section{A. Fast Block Matching with Unimodal Error Surface Assumption}

Most fast block matching algorithms [4]-[6], [13]-[15] restrict the number of search locations using the unimodal error surface assumption, namely, the matching error increases monotonically as the search moves away from the position of the global minimum error. However, this assumption usually does not hold, and as a result, the search could be trapped to a local minimum with a relatively large matching error. Moreover, these algorithms treat each block independently and tend to result in a noisy motion field and create the blocking effect in reconstructed images. Some well-known algorithms in the class include the three-step search (TSS) [6] and the two-dimensional logarithmic search (TDL) [5].

\section{B. Fast Block Matching with Pixel Subsampling}

Another interesting technique to reduce the complexity of MV estimation is block matching with pixel subsampling proposed by Liu and Zaccarin [9]. Instead of limiting the number of search locations, the number of pixels used in matching error computation is reduced. The technique is called alternating 4:1 pixel subsampling in [9], since there are four possible $4: 1$ pixel subsampling patterns to be used alternatively. It was shown that using all four patterns in a specific alternating manner gives a better result than using only one 4:1 subsampling pattern. This technique reduces the number of matching operations by a factor of four. Furthermore, two other techniques were also presented in [9] to enhance the performance. One is called subsampled motion-field estimation which exploits the idea of block subsampling, and the other is called subblock motion-field estimation where a smaller block size is used. The first one reduces the number of operations by a factor of two while the second one has a reduction factor of four. Finally, two fast algorithms based on the combination of the first (alternating $4: 1$ pixel subsampling) and the second (subsampled motionfield) techniques and the combination of the first and the third (sub-block motion-field) techniques were proposed. They reduce the computational complexity by factors of 8 and 16, respectively. The above three techniques provide a set 
of good tools which can be incorporated in several existing algorithms to obtain an additional amount of computational reduction.

\section{Fast Block Matching with Spatial/Temporal Correlations}

Another direction for fast MV estimation approach is to exploit information from adjacent blocks by using spatial and temporal correlations of MV's [18], [20]. The main idea is to select a set of initial MV candidates from spatially and/or temporally neighboring blocks and choose the best one (according to a certain rule) as the initial estimate for further refinement. Theoretically, the initial estimate can be obtained by using an autoregressive (AR) model [18], [20]. For such a simple model, only one candidate is chosen and used as the initial estimate in experiments. The refinement process involves a full search with a reduced search area. It turns out that the full search procedure still requires a considerable amount of computation despite being performed on the reduced search area.

A hybrid algorithm which uses both block-recursive and block-matching methods was proposed in [17]. Although its original motivation did not aim at the use of spatial and temporal correlations, it did provide an interesting way to use both correlations effectively. In the algorithm, the MV candidates were selected from two spatially and one temporally neighboring blocks. In the refinement process, a block recursive idea was explored to compute the gradient direction for MV update. However, the gradient approach does not work well for real-world fast motion image sequences since an oscillation in the search direction may occur in refinement. It is therefore limited to applications with relatively slow motion, e.g. videoconferencing.

\section{Hierarchical and Multiresolution Fast Block Matching}

One family of fast block motion estimation algorithms relies on the idea of predicting an approximate large-scale MV in a coarse-resolution video and refining the predicted MV in a multiresolution fashion to obtain the MV in the finer resolution. They are called the hierarchical [1], [2] or the multiresolution methods [7], [16], [19], [21]. The hierarchical methods [1], [2] use the same image size but different block sizes at each level. The underlying assumption is that the MV obtained from a larger block size provides a good initial estimate for MV's associated with smaller blocks which are contained by the larger block. This assumption is often not true and the estimate can be very poor. Furthermore, a larger block size implies a higher computational cost in performing block matching. The multiresolution methods [7], [16], [19], [21] use different image resolutions with a smaller image size at a coarser level (i.e., of a pyramid form). They can be further divided into two groups: constant block size and variable block size.

In [7] and [16], the same block size is used at each level. Thus, a block at the coarser level represents a larger region than that at the finer level so that a smaller search area can be used at coarser levels. If the image size is reduced by half as the level becomes coarser, one block at a coarser level covers four corresponding blocks at the next finer level. Then, the MV of the coarser-level block is either directly used as the initial estimate for the four corresponding finer-level blocks [7] or interpolated to obtain four MV's of the finer level [16]. In [19] and [21], different block sizes are employed at each level to maintain a one-toone correspondence between blocks in different levels. Then, the MV of each block is directly used as an initial estimate for the corresponding block at the finer level. Methods in this category work relatively well and provide fast computation. However, they only use the information from coarser levels for the MV refinement in finer levels without considering other useful information such as spatial and temporal correlations among MV's at the same level. Furthermore, the refinement process is performed by using a full search algorithm with a reduced search area which nevertheless requires a considerable amount of computation.

\section{E. Overview of Our Work}

Even though many fast MV estimation techniques have been proposed as reviewed before, we feel that the spatial and temporal correlations of MV's have not yet been fully exploited in reducing the search time while maintaining a reasonable rate-distortion tradeoff. The use of an AR model to characterize spatio-temporal correlations of the motion field could provide an elegant theoretical result. However, its derivation requires a certain amount of computational complexity and its practical value decreases. Our goal is to develop a sequence of fast MV estimation algorithms which exploit the spatio-temporal correlations of MV's in a computationally simple way and yet work effectively in the sense of producing small residual errors. Furthermore, we incorporate such correlations in a multiresolution framework to improve the overall performance.

This paper is organized as follows. We first propose two new algorithms using only the spatial correlation. They are called S1 and S2 (where S denotes spatial) and are introduced in Sections II and III, respectively. Algorithm S1 provides the basic framework while algorithm S2 is a modified version. To achieve a better performance, we incorporate the information from the temporal domain and propose two fast algorithms based on spatio-temporal correlations. They are called ST1 and ST2 (where ST stands for spatial and temporal) and are described in Sections IV and V, respectively. Then, we integrate the spatio-temporal technique with the multiresolution scheme to obtain the ultimate algorithm called MRST (where MR denotes multiresolution) in Section VI. The MV's obtained from all proposed algorithms are compatible with the MPEG standard. The performance of all algorithms is demonstrated via extensive experiments in Section VII. Concluding remarks are given in Section VIII.

\section{Fast Algorithm Based on Spatial Correlation: S1}

The following framework is adopted in our discussion. Each image frame is divided into nonoverlapping square blocks of $16 \times 16$ pixels as specified by MPEG. We use $B(i, j, k)$ to represent a block of the $k$ th frame, where $i$ and $j$ are block 
indexes along the row and column directions, respectively. For example, an image of size $352 \times 240$ pixels has block indexes $i=0,1, \cdots, 14$ and $j=0,1, \cdots, 21$, and $B(0, j, k)$ and $B(i, 0, k)$ represent the blocks in the first row and first column, respectively. We would like to determine the MV for each block between two consecutive frames with a certain fast MV estimation algorithm. Without loss of generality, when we talk about the MV of block $B(i, j, k)$, it is calculated based on forward prediction, i.e., the MV between frames $k-1$ and $k$. However, the same idea can be applied to backward prediction and generalized to bidirectional prediction.

It has been observed that the MV of a certain block is the same or very close to the MV's of its spatially adjacent blocks. To illustrate the spatial correlation of MV's, we compute the MV spatial differentials of consecutive blocks along the horizontal and vertical directions via full search for 30 frames of the "football" sequence (frame 31 to 60), and plot the histograms of the averaged occurrence number of $x$ - and $y$ components of these differentials in Fig. 1. The high peaks at the zero differential value indicate that the MV field is highly correlated along both horizontal and vertical directions. We also observe similar histogram plots for other frames in any other image sequences. Such an observation suggests that the MV of a given block can be predicted from its spatially neighboring blocks. This is the main idea behind our algorithm.

Based on this spatial correlation property of MV's, we propose a fast MV estimation algorithm called S1 in this section. It consists of two major building elements: 1) the MV candidate selection and 2) the MV refinement process. Before a detailed discussion on each component, we would like to define the following terms to make the discussion clear.

- The initial MV candidates represent a set of MV candidates selected from spatial (and temporal as well as hierarchical in later sections) neighboring blocks with a certain selection rule.

- The best MV candidate represents the one MV chosen from the set of initial MV candidates to serve as the starting point for the MV refinement process.

- The final $M V$ represents the final MV result obtained.

\section{A. MV Candidate Selection}

To begin the MV estimation process, we perform the full search block matching algorithm to determine the MV's of the four blocks at the top left corner, i.e., $B(0,0, k), B(0,1, k), B(1,0, k)$, and $B(1,1, k)$. Since the search cannot go beyond image boundaries, MV's of boundary blocks $B(0,0, k), B(0,1, k)$, and $B(1,0, k)$ are limited such that all pixels referenced by them are within the image area, and therefore, a full search at block $B(1,1, k)$ will provide a more accurate initial MV candidate for the following estimation task. When performing a full search, an alternating 4:1 pixel subsampling technique [9] can be used to reduce the computational cost, i.e., for a block of size $16 \times 16$, only the values at 64 pixels are used to compute the mean of absolute difference (MAD). It was shown in [9] that a reasonably good MV estimate can be obtained by using such a pixel

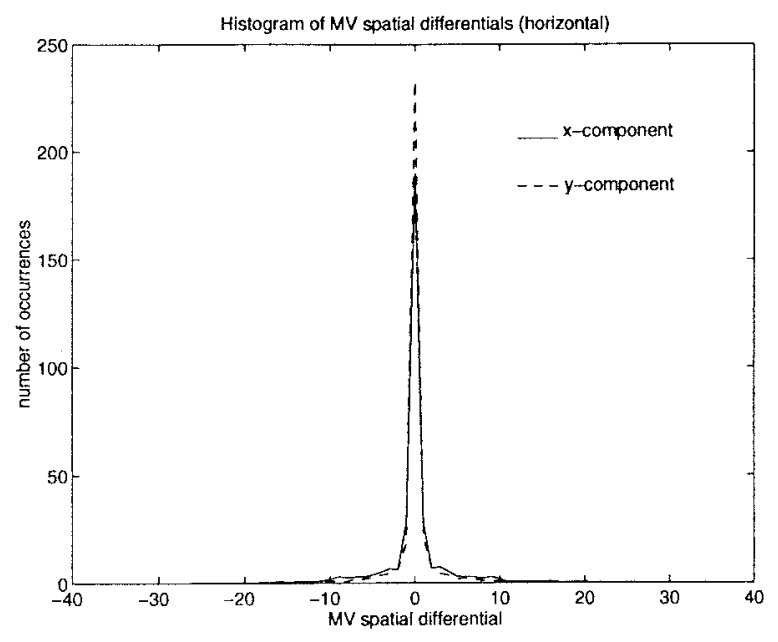

(a)

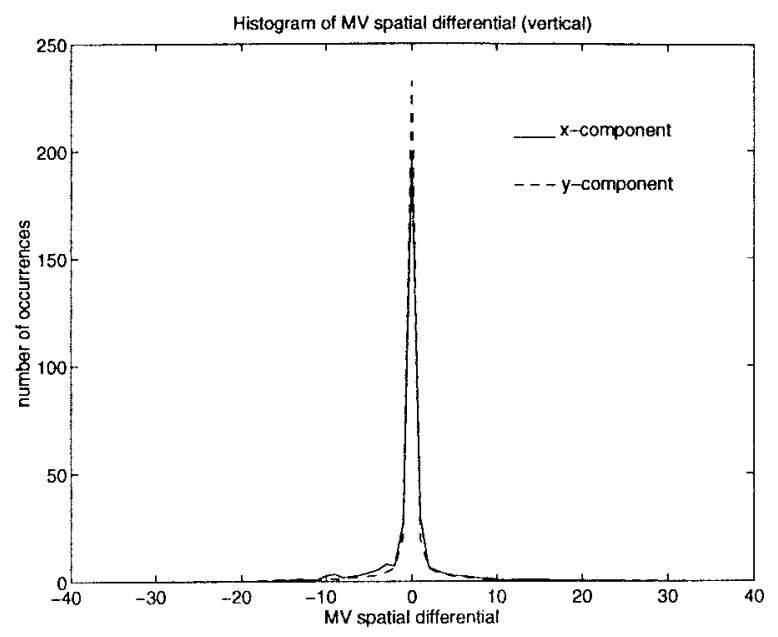

(b)

Fig. 1. Histograms of the averaged number of MV spatial differentials of $\mathrm{x}$ and y-components along the (a) horizontal and (b) vertical directions for 30 frames of the "football" sequence ( $\mathrm{ft} 31$ to $\mathrm{ft} 60)$.

subsampling technique. This gives a computational reduction by a factor of four in comparison with a straightforward implementation of the MAD computation.

After the initialization step, we proceed to the next block according to a rowwise ordering, i.e., starting from the left to the right for the first row, then the second row, and so on, and use the MV's of blocks $B(i, j-1, k), B(i-1, j, k)$, $B(i-1, j-1, k)$, and $B(i-1, j+1, k)$ as the initial MV candidates for block $B(i, j, k)$. Note, however, that blocks along the boundaries have fewer initial candidates than the inner blocks. Among the four initial MV candidates, the one with the smallest MAD (denoted by $M A D_{0}$ ) is chosen as the best MV candidate $\left(V_{0}\right)$ for block $B(i, j, k)$ and used as the starting point for further MV refinement. In other words, let $\mathcal{C}=\left\{C_{1}, \cdots, C_{M}\right\}$ be the set of initial MV candidates, and $M A D\left(C_{i}\right)$ be the MAD corresponding to the vector $C_{i}$. Then, the best MV candidate $V_{0}$ can be expressed as

$$
V_{0}=\arg \min _{C \in \mathcal{C}} M A D(C)
$$




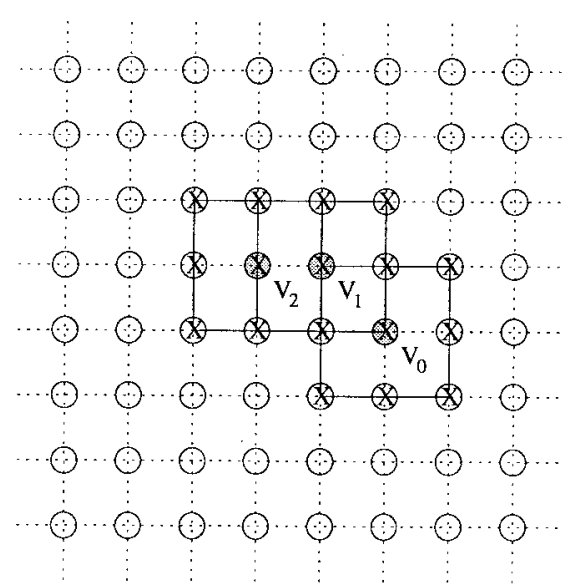

Fig. 2. Local search around procedure: the search starts at the new center (best MV candidate) $V_{0}$ and its eight neighboring points, and moves from $V_{0}$ to $V_{1}$ and to $V_{2}$ since the minimum points in the first two steps are not in the center and the new MAD's are still greater than $T H_{1}$. The search stops after searching around $V_{2}$ since the minimum is located at the center $V_{2}$.

\section{B. MV Refinement Process}

The refinement process begins with the best MV candidate $\left(V_{0}\right)$ and its corresponding MAD value $\left(M A D_{0}\right)$. A threshold $T H_{1}$ is set so that if $M A D_{0} \leq T H_{1}$, the best MV candidate $V_{0}$ is chosen as the final MV for block $B(i, j, k)$. If $M A D_{0}>$ $T H_{1}$, we use the spatial correlation property and assume that the best MV candidate $V_{0}$ is close to the desired final MV. Even though the assumption that the matching error increases monotonically as the searching point moves away from the global minimum (i.e., the unimodal error surface assumption) is generally not true, it seems reasonable to assume that the matching error surface is monotonic in a small neighborhood around the global minimum. This assumption was used to find small motion for low bit-rate coding applications in [8]. It implies that if the initial search point is close to the global minimum, there is a high probability to find the global minimum. In the current context, the best candidate $V_{0}$ is viewed as a new search center so that a search is performed around its neighborhood. The search starts at the new center and its eight neighboring points. If either the minimum MAD among nine of them occurs in the middle (center), or the new smaller MAD has the value $\leq T H_{1}$, the procedure stops. Otherwise, it keeps the same search procedure by using the position with the new minimum MAD as the new search center. The process iterates until either the stopping criterion is satisfied or the preset maximum number $(S)$ of search steps is reached. We call the above search procedure local search around and depict it in Fig. 2. As shown in the figure, eight surrounding locations are searched at each step.

It is observed that the 4:1 pixel subsampling technique gives an unacceptably coarse matching result in the current context, since we consider nine search points at a time and need an accurate local minimum point for the next step. It is nevertheless possible to reduce computation in the MAD calculation at each location. That is, we consider the checkerboard partitioning of $16 \times 16$ pixels within each block and use one half of the pixels beginning at top left pixel for matching. It turns out that this reduction gives sufficiently good results required for a further refinement. Furthermore, even though we have to compute the MAD for nine points at the first step, the MAD has to be computed at only three or five positions in the following steps due to the overlap of the neighborhoods of consecutive centers.

As discussed above, there are three possible conditions for the local search around procedure to stop. First, if it stops because $M A D \leq T H_{1}$, the position corresponding to this MAD value is chosen as the desired final MV. Second, if the procedure stops because of reaching the maximum step number of iteration, it is likely that the MV of the current block may not be close to MV's of its neighboring blocks. This phenomenon often results from motion discontinuity, occluded regions, or a certain type of mixed motion. Thus, we perform a full search with alternating 4:1 pixel subsampling to find the MV for this block. Third, if the search stops because the minimum MAD occurs in the middle among nine points, it seems reasonable to choose that point as the final MV. However, it is possible that the best MV candidate $V_{0}$ is too far from the desired final MV so that we actually get trapped to a wrong local minimum. To avoid such a problem, we check the MAD value at that point. If its MAD value is not larger than a threshold value $\mathrm{TH}_{2}$ (another threshold which is greater than $T H_{1}$ ), then we choose the corresponding position to be the final MV. Otherwise, a full search algorithm with alternating 4:1 pixel subsampling is performed to determine the MV for the current block. This completes our refinement process. Let us summarize Algorithm S1 as follows.

\section{Algorithm S1}

1) The algorithm is initialized by performing a full search with an alternating 4:1 pixel subsampling technique on four blocks located at the top left corner, i.e., $B(0,0, k), B(0,1, k), B(1,0, k)$, and $B(1,1, k)$. The full search algorithm has the maximum displacement $\pm W$ along both horizontal and vertical directions.

2) We proceed the MV search for the block from the top left to the bottom right with a rowwise ordering. For block $B(i, j, k)$, we use the MV's from its four neighboring blocks $B(i, j-1, k), B(i-1, j, k), B(i-1, j-1, k)$, and $B(i-1, j+1, k)$ as possible initial candidates and choose the one with the smallest $\operatorname{MAD}\left(M A D_{0}\right)$ as the best MV candidate $\left(V_{0}\right)$ for the block $B(i, j, k)$.

3) If $M A D_{0} \leq T H_{1}$, the best $\mathrm{MV}$ candidate $V_{0}$ is chosen as the final MV. Then, we move to the next block (returning to Step 2). Otherwise, go to Step 4.

4) If $M A D_{0}>T H_{1}$, perform the local search around procedure until reaching one of the following three stopping criteria:

i) the new minimum $\mathrm{MAD} \leq T H_{1}$;

ii) the minimum MAD among nine points at one step occurs at the middle (center) location;

iii) the search step number reaches the maximum limit $(S)$.

5) If the search stops because of condition i), the position giving the minimum MAD is chosen as the final MV. If the search stops due to condition ii), we check the minimum MAD value in the center. If the MAD value $\leq$ 


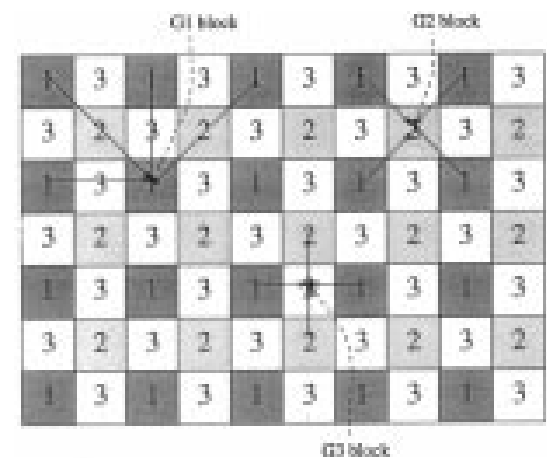

Fig. 3. Block pattern and the illustration of the MV candidate selection procedure for Algorithm S2, where groups $G 1, G 2$, and $G 3$ are denoted by 1,2 , and 3 , respectively.

$\mathrm{TH}_{2}$ (another threshold greater than $T H_{1}$ ), the position is accepted as the final MV. Otherwise, switch to the full search mode with alternating $4: 1$ pixel subsampling to determine the MV. If the search stops because of condition iii), a full search with alternating 4:1 pixel subsampling is performed to obtain the final MV.

6) Proceed to the next block by returning to Step 2. After all the blocks in a frame are processed, go to the next frame with Step 1.

\section{MOdIFIED SPATIAL-BASED AlgORITHM: S2}

The spatial correlation of MV's was exploited to develop a fast MV estimation algorithm known as S1 in Section II. In this section, we propose another algorithm called S2 with the objective to speed up the search computation while maintaining a similar performance in the resulting MSE (or ratedistortion). The new algorithm is motivated by the observation that, in many cases, the MV's have a long range spatial correlation. That is, the MV's associated with 2: 1 horizontally or vertically subsampled blocks still have a strong spatial correlation. Thus, by using block subsampling, we classify blocks into several different types. Then, the MV of a certain type of block can have its initial MV candidates coming from four noncausal spatial directions rather than causal directions (i.e., upper and left) only as occurred in S1. The resulting scheme provides a faster search for these blocks since a better set of initial MV candidates is used. Consequently, we obtain an overall performance improvement.

\section{A. MV Candidate Selection}

We classify each block into one of the three types $G 1$, G2, and $G 3$ as shown in Fig. 3. We first treat the image as if it consists only of $G 1$ blocks, and perform the MV estimation for blocks in the $G 1$ group with Algorithm S1. That is, for block $B(i, j, k)$ in $G 1$, we use the MV's from blocks $B(i, j-2, k), B(i-2, j, k), B(i-2, j-2, k)$, and $B(i-2, j+2, k)$ to be initial MV candidates as shown in Fig. 3, and the one with the smallest MAD is chosen as the best MV candidate for further MV refinement to obtain the final MV.

Each $G 2$ block is surrounded by four $G 1$ blocks at its four corner positions. We use the MV's from the four $G 1$ blocks as initial MV candidates and choose the one with the smallest MAD as the best MV candidate for further refinement. There are some $G 2$ blocks which lie along image boundaries and require some special attention. Following the above rule, its initial MV candidates come from one or two nonboundary blocks. However, the boundary blocks can only have a certain range of MV's so that their MV's may be quite different from those of the inner blocks. Thus, we include the MV from the nearest $G 2$ block located on the same boundary which has been obtained earlier as an additional initial MV candidate for boundary $G 2$ blocks. Finally, we perform the MV refinement process to obtain the final MV for $G 2$ blocks.

The next step is to estimate the MV's for $G 3$ blocks. As shown in Fig. 3, we now have the initial MV candidates from $G 1$ and $G 2$ blocks. To be more precise, for a $G 3$ block $B(i, j, k)$, we use the four MV's from blocks $B(i, j-$ $1, k), B(i, j+1, k), B(i-1, j, k)$, and $B(i+1, j, k)$ as its initial MV candidates. These MV candidates come from blocks located at the same row or the same column, and they tend to provide a better candidate set than the ones provided by four corner blocks. Blocks along boundaries can also be handled in a straightforward fashion. The best MV candidate is obtained from the one giving the smallest MAD and used for the further refinement.

\section{B. MV Refinement Process}

The refinement process for blocks in groups $G 1$ and $G 2$ is performed in the same way as that in S1 (see Section II-B) with a slight modification for $G 3$ blocks. As mentioned above, G3 blocks tend to have a better set of initial MV candidates than $G 1$ and $G 2$ blocks so that the MV of each $G 3$ block should be close to one of its surrounding initial MV candidates. Thus, the refinement for $G 3$ blocks is performed without switching to the full search mode. In other words, after choosing the best $\mathrm{MV}$ candidate $\left(V_{0}\right)$ with the smallest MAD among initial $\mathrm{MV}$ candidates, if $M A D_{0} \leq T H_{1}$, the vector $V_{0}$ is chosen to be the final MV. Otherwise, we perform the local search around. Whatever condition stops the search, we accept the position with the last minimum MAD as the final MV.

In the next section, we will show how to incorporate the information from the temporal direction to improve the performance.

\section{FAST AlgORITHM BASED ON SPATIO-TEMPORAL CORRELATION: ST1}

In addition to the spatial correlation, MV's are also highly correlated in the temporal direction. We observe that the histogram of MV temporal differentials is similar to that of MV spatial differentials as shown in Fig. 1 with a high peak appearing at the zero differential value. The temporal correlation property provides additional information for selecting a better set of initial MV candidates and leads to an overall improvement in the search speed and the resulting MSE. In this section, we modify Algorithm S1 by including temporal information. The resulting algorithm is called Algorithm ST1.

A simple way to incorporate the temporal information in Algorithm S1 is to include the MV of the block at the same 
location from the previous frame (i.e., $B(i, j, k-1)$ ) in the set of initial MV candidates. Furthermore, we can obtain information from noncausal directions by including two more initial MV candidates obtained from the blocks in the previous frame, i.e., $B(i+1, j, k-1)$ and $B(i, j+1, k-1)$. Finally, it is natural to include MV's of $B(i-1, j, k)$ and $B(i, j-1, k)$. Thus, for block $B(i, j, k)$, there are five initial MV candidates from blocks

$$
\begin{gathered}
B(i, j-1, k), \quad B(i-1, j, k), \quad B(i, j, k-1), \\
B(i, j+1, k-1), \quad B(i+1, j, k-1)
\end{gathered}
$$

except for the first predicted frame where the temporal information is not available and Algorithm S1 can be performed to find the MV's.

Note that the basic idea of choosing the initial MV candidates from the above five blocks is to obtain the information from all surrounding directions as well as from different block locations. For example, blocks $B(i, j-1, k)$ and $B(i-1, j, k)$ are selected from the current frame, therefore, we do not choose the same block locations, i.e., $B(i, j-1, k-1)$ and $B(i-1, j, k-1)$ from the previous frame. Even though there are more than five vectors available to serve as the initial MV candidates, we should not select too many vectors due to the additional computation required in calculating the MAD value for each vector.

Recall that we initialize the MV estimation process in S1 by performing a full search for the four blocks at the upper left corner by exploiting the spatial correlation only. By including temporal information, we can use temporal information to select initial MV candidates for these four blocks without full search in initialization. After selecting a set of initial MV candidates, we choose the one giving the smallest MAD value $\left(M A D_{0}\right)$ as the best MV candidate $\left(V_{0}\right)$ and perform further refinement to improve the MV result. The refinement process is performed in exactly the same way as stated in Section II-B.

\section{Modified Spatio-Temporal Based Algorithm: ST2}

With the framework of the modified algorithm S2, we propose the new spatio-temporal correlation based algorithm ST2 by incorporating the temporal correlation information to improve the speed of Algorithm ST1.

In Algorithm ST2, each block is assigned to one of three groups in the same pattern as in Algorithm S2 (Fig. 3). For $G 1$ blocks in Algorithm S2, the only available initial MV candidates come from spatially subsampled blocks in causal (upper left) directions. In ST2, we include candidates from blocks in lower, right, and the same locations from the previous frame. Thus, for block $B(i, j, k)$ in the $G 1$ group, we select the MV's from two spatially adjacent blocks $B(i, j-2, k)$ and $B(i-2, j, k)$, and three temporally adjacent blocks $B(i, j, k-1), B(i, j+1, k-1)$, and $B(i+1, j, k-1)$ to be initial MV candidates as shown in Fig. 4. Note that there is no need to perform full search for the initialization of $G 1$ blocks at the upper left corner due to availability of temporal information. The best MV candidate is obtained by choosing the one with the smallest MAD. Then, we perform further refinement.

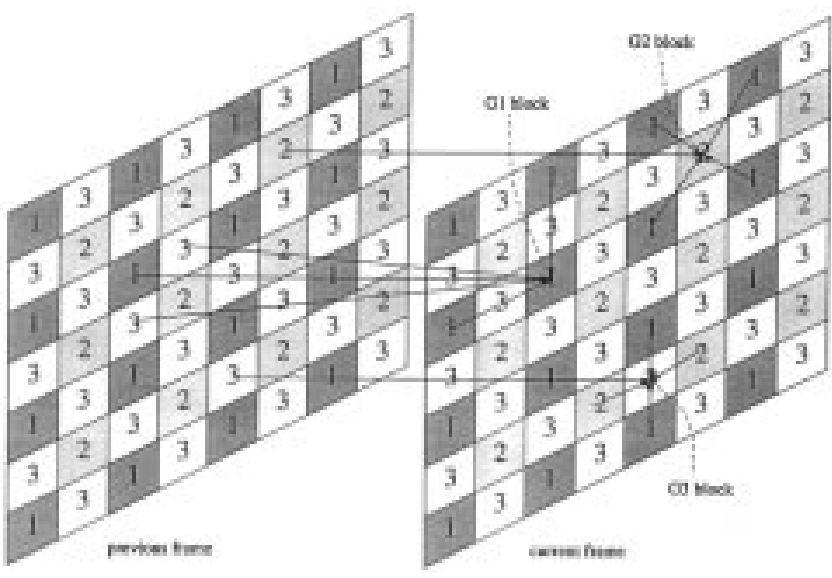

Fig. 4. The MV candidate selection procedure for Algorithm ST2.

The $G 2$ blocks have more MV information available for their spatially neighboring blocks than $G 1$, since each $G 2$ block is surrounded by four $G 1$ blocks at its four corner positions. We use the MV's from four spatial $G 1$ blocks and one temporal $G 2$ block on the same location from the previous frame as initial MV candidates as shown in Fig. 4.

Similar to Algorithm S2, G3 blocks have initial MV candidates from $G 1$ and $G 2$ blocks which are located in either the same row or column. Thus, for a $G 3$ block $B(i, j, k)$, we use the four MV's from spatial blocks $B(i, j-1, k), B(i, j+$ $1, k), B(i-1, j, k)$, and $B(i+1, j, k)$ and one MV from temporal block $B(i, j, k-1)$ as its initial MV candidates. The selection rule is shown in Fig. 4.

Note that each $G 2$ or $G 3$ block is surrounded by four spatial candidates with one temporal candidate in the middle location. Such a candidate pattern suggests a simple modification on the decision rule. That is, if all five initial candidates are the same, such a vector can be used as the final MV without any further refinement. This modification saves a certain amount of MAD computation with little sacrifice in the performance. If they are not the same, we employ the original rule where the best MV candidate is set to the candidate with the smallest MAD. Then, we perform the same local refinement as that in Algorithm S2 for each group.

The proposed MV selection procedure of ST2 provides a scheme to use spatial and temporal information in a complement way. For example, $G 1$ blocks do not have as many good spatial candidates as the $G 2$ and $G 3$ blocks do, therefore, they need more information from the temporal direction. For $G 2$ and $G 3$ blocks, we give a higher priority to noncausal information from the spatial domain than the temporal domain as reflected from the fact that four (noncausal) spatial neighboring MV's and one temporal MV are used for $G 2$ and G3 blocks.

We have seen so far that all proposed algorithms S1, S2, ST1, and ST2 require threshold parameters $T H_{1}$ and $\mathrm{TH}_{2}$. These parameters are preset as certain fixed numbers. However, it would be desirable if such parameters can be chosen or adjusted automatically from the algorithm itself. In the next section, we propose a new fast algorithm based on multiresolution-spatio-temporal correlations, which not only 
solves the threshold problem but also improves the overall performance.

\section{FAST MV ESTIMATION USING MULTIRESOLUTION-SPATIO-TEMPORAL CORRELATIONS: MRST}

The multiresolution approach has been recently studied and applied to motion estimation problem [7], [16], [19], [21]. It provides a relatively fast computational speed while giving a reasonably good prediction. With this approach, an image frame is decomposed into different resolutions. The multiresolution nature provides a hierarchical motion field obtained at different scales, and a smaller search area is used at a coarser level. The MV estimation is first performed on the coarsest resolution, and then the MV's of finer resolutions are refined based on the motion information obtained at coarser resolutions. Most existing algorithms use only information from coarser levels to refine the MV in finer levels and do not exploit spatio-temporal correlations of the MV's at the same level. In the section, we propose a new fast algorithm called MRST by combining the multiresolution, spatial, and temporal correlation properties.

In this work, a coarser resolution image is obtained by computing the mean of $2 \times 2$ pixels from finer levels to represent a pixel in the next coarser level so that the image size is reduced by half along both horizontal and vertical directions. Note that since we focus on the MV estimation problem without worrying about the residual coding, only a simple averaged mean is used here to obtain coarser resolution images. We also employ different block sizes at different levels as presented in [19] and [21], and blocks of smaller sizes are used in the coarser levels so that each level has the same number of blocks. Thus, there is a one-to-one correspondence of blocks between coarser and finer levels. The MV's at different levels are highly correlated since they represent the same motion activities at different scales.

In the proposed algorithm MRST, each image frame is decomposed into four resolution levels with a block of size $2 \times 2$ at the coarsest level and a block of a size $16 \times 16$ at the finest level. The level numbers are ordered from 0 to 3 , where levels 0 and 3 represent the coarsest and finest levels, respectively. We begin with the coarsest level by performing a full search to obtain the MV for each block. Due to the coarse scale of the MV, the maximum search displacement at the coarsest level is reduced from $\pm W$ to $\pm W / 8$ so that only a small amount of computation is required.

For each of the finer resolution levels (levels 1 to 3), we adopt the framework of Algorithm ST2 (except for the first predicted frame for which Algorithm S2 is applied) by using the MV information from the coarser level as well as spatially and temporally neighboring blocks at the same level. Most existing algorithms rely only on the initial information from the corresponding coarse-scaled MV's for further refinement. However, the coarse-scaled MV's for some blocks may not be accurate enough and could cause some errors which propagate along the hierarchical structure. Our algorithm exploits the information from both multiresolution and spatio-temporal adjacent blocks to select a number of initial MV candidates. Note that the MV from the coarser level has to be properly

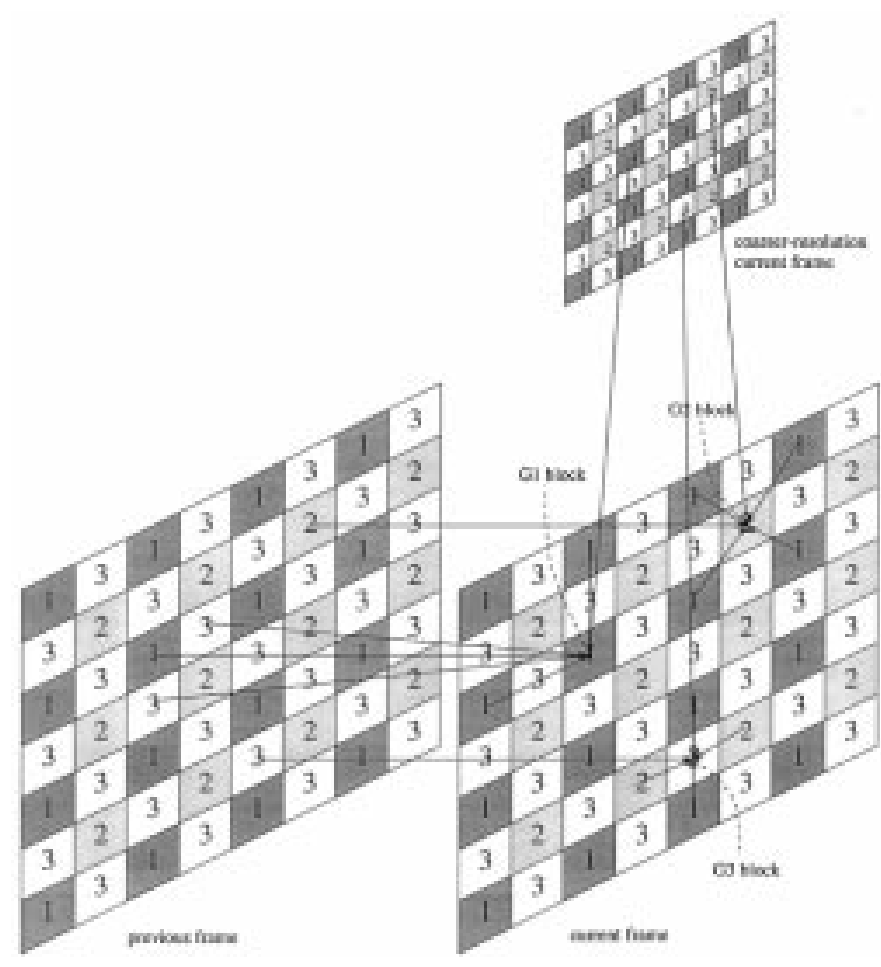

Fig. 5. The MV candidate selection procedure for Algorithm MRST.

scaled to serve as the initial candidate in the finer level. For example, if a block at level $l$ has the motion vector $V$, then the corresponding block at level $l+1$ uses $2 V$ as its initial MV candidate. The set of initial MV candidates can be expressed as

$$
\left\{C_{1}, C_{2}, \cdots, C_{M}, C_{M+1}\right\}
$$

where $C_{1}, \cdots, C_{M}$ are initial MV candidates obtained from the MV selection procedure specified in ST2 and $C_{M+1}$ is the initial candidate from the corresponding coarser level. Since the available temporal MV candidate is the final predicted MV from the previous frame (i.e., MV with the finest resolution), the vector has to be scaled in order to be used as the initial candidate in the middle levels. That is, let $V_{t}$ denote the temporal MV candidate in the finest level 3. We use the vector $V_{t} / 2^{3-l}$ with a proper truncated value as the initial candidate for levels $l=1,2$ (middle levels). A graphic explanation of the MV selection procedure for Algorithm MRST is illustrated in Fig. 5.

The best MV candidate is obtained by using the same rule as that described in ST2. Similar to Algorithm ST2, we also adopt a majority checking rule to select best MV candidate for $G 2$ and $G 3$ blocks. According to the above selection process, each $G 2$ or $G 3$ block has a total of six candidates (four from spatial, one from temporal, and one from the coarser level). The new checking rule is that if at least five candidates are the same, this value is the final MV and no further refinement is required. This allows us to save a certain amount of computation with little sacrifice in MSE increase.

Note that when the MV information from the coarse level is used, we can only have the MV of an even number of length as the initial candidate. On the other hand, if only the spatio-temporal information is used as in ST2, there are some 
blocks that still require a full search (those having the MAD greater than $\mathrm{TH}_{2}$ or reaching the maximum iteration limit). By incorporating the coarser level information, the MV obtained from full search in the coarsest level provides a better set of candidates so that the full search mode is not needed at finer levels. The new algorithm is more robust in the sense that the second threshold $\mathrm{TH}_{2}$ is no longer needed.

One important issue in the refinement process is the selection of threshold $T H_{1}$. It is one main factor to determine the computational speed since it indicates whether to choose the best $\mathrm{MV}$ candidate as the final MV (according to the criterion $\left.M A D_{0} \leq T H_{1}\right)$. In Algorithms S1, S2, ST1, and ST2, the threshold $T H_{1}$ is a fixed number for every frame throughout the entire estimation process. It would be more desirable to find a rule such that $T H_{1}$ can be adjusted adaptively for different frames to provide a higher computational speed. Such a rule should be related to the knowledge of matching error (MAD) information. In Algorithm MRST, we obtain the values of minimum MAD's via full search at the coarsest level. Such values should give us some rough idea about the range of smallest MAD's for each frame. Therefore, we compute the averaged mean of smallest MAD's over all blocks at the coarsest level for each frame (denoted as $u_{k}$, where $k$ is the frame number) and use it to determine the value of $T H_{1}$. We also observe that $u_{k}$ tends to have a small increase as the resolution level goes finer for most frames. Based on the observation, we choose the threshold value $T H_{1}$ by adding 0.5 to $u_{k}$ every time as the level becomes finer, i.e.,

$$
T H_{1}(l)=u_{k}+(0.5 \times l)
$$

at level $l$. Although we know the smallest MAD of each block at the coarsest level, there is no direct relationship among the smallest MAD's of blocks at different levels. Therefore, it is difficult to adopt any specific rule for $T H_{1}$ value of each block separately since it could give a large error to some blocks. Instead, we use the averaged mean value as described above so that the threshold $T H_{1}$ is good enough in the average sense and would not produce a significantly large error. Based on the above rule, threshold $T H_{1}$ is automatically chosen from the MAD information of the coarsest level. The new algorithm MRST is summarized as follows.

\section{Algorithm MRST}

1) Obtain the coarser resolution images by computing the averaged mean of the nonoverlapping $2 \times 2$ pixels from finer-level images for each pixel in coarser-level images until the block size at the coarsest level becomes 2 $\times 2$. Let the total number of resolution levels be $L$. The image at resolution $l$ is divided into blocks of size $2^{l-L+1} B \times 2^{l-L+1} B$, where $B(=16)$ is the block size of the finest resolution $L-1$ and $l=0,1, \cdots, L-1$. ( $L=4$ in the current case).

2) At the coarsest level, perform a full search with the maximum search displacement $\pm\left(W / 2^{L-1}\right)$ to obtain the MV for each block.

3) For each finer level, perform Algorithm ST2 (except for the first predicted frame for which S2 is applied) by including one more candidate from the corresponding block in the previous coarser level by multiplying the
TABLE I

Experimental Results of Five Test Video Sequences with the Log Search and the Full SEarch

\begin{tabular}{l||c|c|c||c|c}
\hline \multicolumn{1}{c||}{ Video } & \multicolumn{3}{c||}{ Log search } & \multicolumn{2}{c}{ FBMA } \\
\cline { 2 - 6 } & $\begin{array}{c}\text { Speed- } \\
\text { up }\end{array}$ & $\begin{array}{c}\text { MSE } \\
\text { (\% increase) }\end{array}$ & $\begin{array}{c}\text { MV } \\
\text { bits }\end{array}$ & MSE & $\begin{array}{c}\text { MV } \\
\text { bits }\end{array}$ \\
\hline Bicycle & 48.6 & $535.9(27.7 \%)$ & 2695 & 419.5 & 2405 \\
\hline Cheer & 56.4 & $542.6(16.9 \%)$ & 1856 & 464.3 & 2121 \\
\hline Flower & 54.0 & $250.1(13.2 \%)$ & 1297 & 221.0 & 1080 \\
\hline Football & 45.6 & $231.3(32.5 \%)$ & 3149 & 174.6 & 2450 \\
\hline Mobile & 62.6 & $349.0(7.0 \%)$ & 893 & 326.3 & 840 \\
\hline
\end{tabular}

coarser-scaled MV by a factor of two. The temporal MV candidates have to be properly scaled as well. The refinement process is performed in the same way as done in ST2 except the adoption of full search in any case for any block type ( $G 1, G 2$, or $G 3)$.

4) After the MV's at finest level are obtained, move to the next frame beginning with Step 1.

\section{EXPERIMENTAL RESULTS}

Experimental results using all proposed algorithms S1, S2, ST1, ST2, and MRST are reported in this section. They are applied to five MPEG test videos: bicycle, cheer (leaders), flower, football, and mobile. Each video contains 150 frames and each frame has a size of $352 \times 240$ pixels obtained by subsampling CCIR601 $720 \times 480$ luminance component only. These sequences provide a variety of motions including slow and fast movements (cheer and football), camera panning (flower and mobile), and zooming (bicycle). A $16 \times 16$ square block is used as a macroblock for MV estimation as specified by MPEG. Only forward prediction is implemented in the experiments. The maximum horizontal and vertical search displacement is \pm 16 ( $W=16$ ) so that the maximum search locations for one block is $33 \times 33(33=(2 \times 16)+1)$. We use MAD as the error measure in performing block matching, while the MSE per pixel is computed between the original and the resulting motion-compensated frames as the quality measure. For Algorithms S1, S2, ST1, and ST2, the threshold parameters used are $T H_{1}=4$ and $T H_{2}=35$, and the maximum step number for local search around is $S=10$. Algorithm MRST does not require $T H_{1}$ and $T H_{2}$, and the maximum search step number for each finer level is two.

Note that the values of threshold parameters $\mathrm{TH}_{1}$ and $\mathrm{TH}_{2}$ are empirical numbers. Although their optimal values should be different for various sequences, we use the same values for all sequences to show the robustness of our algorithms. That is, by using fixed threshold values, our algorithms still give reasonably good results for all test sequences. As mentioned in the previous section, the problem of selecting a proper threshold value can be completely avoided when using Algorithm MRST, which is our ultimate algorithm.

For comparison, we show in Table I the results obtained from the FBMA and the two-dimensional (2-D) logarithmic search [5]. To demonstrate the performance of our algorithms, we present various results with all five proposed algorithms in Tables II-VI. All values in the tables are the averaged 
values by using data obtained from 149 predicted frames. The meaning of each column is explained below.

- Speed-up represents the speed-up factor gained from the algorithms. To be more precise, a speed-up factor $=P$ means that the matching operations are reduced by a factor of $P$ from full search. For example, with \pm 16 search displacement in the full search case, each block of size $16 \times 16(=256)$ pixels is searched for $33 \times$ 33 (=1089) locations in order to obtain the MV. Thus, there are $256 \times 1089$ operations in total for each block. Let us say that in our proposed algorithm, if we use only $50 \%$ of pixels (128 pixels) in a block and search for $N$ locations, then the number of operations is $128 \times N$, and the corresponding speed-up factor is $2 \times 1089 / N$ for such a block. However, since we have a total of 330 blocks, we need to add the number of operations together for all blocks before computing the speed-up factor.

- MSE (\% increase) represents the increased MSE of reconstructed motion-compensated frames with the MSE obtained via full search as the reference, which is expressed in terms of percentage.

- Search step represents the averaged step number in performing the local search around (presented in Tables II-V). This includes step number 0 with $M A D_{0} \leq$ $\mathrm{TH}_{1}$ (i.e., the best $\mathrm{MV}$ candidate is used as the final MV without MV refinement).

- FS bks shows the averaged number of blocks requiring full search for each frame (presented in Tables II-V).

- $M V$ bits represents the number of bits used in the coding of MV's, where the differential coding scheme adopted by the MPEG standard [10] is applied.

By comparing Algorithms S1 and ST1, and Algorithms S2 and ST2, we can see a significant improvement in the speed-up factor by using both spatial and temporal correlations rather than using the spatial correlation only. Furthermore, we see from Tables II-V that the averaged speed-up factors of ST2 (or S2) are higher than those of ST1 (or S1) in all five sequences. The main reason for gaining a high speed-up factor in ST2 (or S2) is due to computational reduction in $G 2$ and $G 3$ blocks, especially in $G 3$ blocks where the number of blocks counts for one half of the total number of blocks. In Table VI, we see that the speed-up factors of MRST are higher than those of S1, S2, ST1, ST2, and log search. Due to multiresolution and spatio-temporal correlations, we can obtain a better set of initial MV candidates so that there is no need to perform full search at finer levels (no $\mathrm{TH}_{2}$ ). Note, however, that for "mobile" sequence, MRST gives a little lower speed-up factor than ST2 with a similar MSE result. This is because the MV's of "mobile" sequence have very strong spatial and temporal correlations so that very few blocks require full search in ST2 (only the average of 0.7 block in "FS bks" column). For this case, multiple levels in MRST seem to add a little more computation which causes a lower speed-up factor than that of ST2. For "cheer" and "football" sequences which have a larger number of "FS bks," we see that Algorithm MRST results in a much higher speed-up factor than ST2.
TABLE II

EXPerimental Results of Five Test Video Sequences with Algorithm S1

\begin{tabular}{l||c|c|c|c|c}
\hline \multicolumn{1}{c||}{ Video } & \multicolumn{5}{c}{ Proposed algorithm S1 } \\
\cline { 2 - 6 } & $\begin{array}{c}\text { Speed- } \\
\text { up }\end{array}$ & $\begin{array}{c}\text { MSE } \\
\text { (\% increase) }\end{array}$ & $\begin{array}{c}\text { Search } \\
\text { step }\end{array}$ & $\begin{array}{c}\text { FS } \\
\text { bks }\end{array}$ & $\begin{array}{c}\text { MV } \\
\text { bits }\end{array}$ \\
\hline Bicycle & 74.3 & $458.8(9.4 \%)$ & 1.81 & 11.9 & 1946 \\
\hline Cheer & 55.1 & $495.6(6.7 \%)$ & 1.57 & 19.4 & 1904 \\
\hline Ilower & 110.2 & $226.7(2.6 \%)$ & 0.87 & 7.3 & 957 \\
\hline Football & 83.1 & $205.3(17.6 \%)$ & 1.51 & 10.8 & 1991 \\
\hline Mobile & 128.4 & $330.6(1.3 \%)$ & 0.87 & 5.6 & 807 \\
\hline
\end{tabular}

TABLE III

Experimental Results of Five Test Video Sequences with Algorithm S2

\begin{tabular}{l|c|c|c|c|c}
\hline \multirow{2}{*}{ Video } & \multicolumn{5}{|c}{ Proposed algorithm S2 } \\
\cline { 2 - 6 } & $\begin{array}{c}\text { Speed- } \\
\text { up }\end{array}$ & $\begin{array}{c}\text { MSE } \\
\text { (\% increase) }\end{array}$ & $\begin{array}{c}\text { Search } \\
\text { step }\end{array}$ & $\begin{array}{c}\text { FS } \\
\text { bks }\end{array}$ & $\begin{array}{c}\text { MV } \\
\text { bits }\end{array}$ \\
\hline Bicycle & 85.8 & $463.6(10.5 \%)$ & 1.81 & 8.2 & 1934 \\
\hline Cheer & 70.6 & $512.4(10.3 \%)$ & 1.57 & 13.0 & 1863 \\
\hline Flower & 128.9 & $228.6(3.4 \%)$ & 0.85 & 5.5 & 981 \\
\hline Football & 95.2 & $207.9(19.1 \%)$ & 1.50 & 8.4 & 2039 \\
\hline Mobile & 138.0 & $331.5(1.6 \%)$ & 0.86 & 4.8 & 802 \\
\hline
\end{tabular}

TABLE IV

EXPERIMENTAL Results of Five Test Video Sequences with Algorithm ST1

\begin{tabular}{l||c|c|c|c|c}
\hline \multicolumn{1}{c||}{ Video } & \multicolumn{5}{c}{ Proposed algorithm ST1 } \\
\cline { 2 - 6 } & $\begin{array}{c}\text { Speed- } \\
\text { up }\end{array}$ & $\begin{array}{c}\text { MSE } \\
\text { (\% increase) }\end{array}$ & $\begin{array}{c}\text { Search } \\
\text { step }\end{array}$ & $\begin{array}{c}\text { FS } \\
\text { bks }\end{array}$ & $\begin{array}{c}\text { MV } \\
\text { bits }\end{array}$ \\
\hline Bicycle & 110.7 & $450.1(7.3 \%)$ & 1.56 & 5.7 & 1997 \\
\hline Cheer & 84.4 & $489.9(5.5 \%)$ & 1.31 & 11.4 & 1911 \\
\hline Flower & 178.1 & $224.6(1.7 \%)$ & 0.75 & 3.0 & 954 \\
\hline Football & 151.5 & $195.2(11.8 \%)$ & 1.24 & 3.7 & 2034 \\
\hline Mobile & 217.5 & $330.4(1.3 \%)$ & 0.80 & 1.2 & 808 \\
\hline
\end{tabular}

TABLE V

Experimental Results of Five Test Video Seouences with Algorithm ST2

\begin{tabular}{l||c|c|c|c|c}
\hline \multicolumn{1}{c||}{ Video } & \multicolumn{5}{c}{ Proposed algorithm ST2 } \\
\cline { 2 - 7 } & $\begin{array}{c}\text { Specd- } \\
\text { up }\end{array}$ & $\begin{array}{c}\text { MSF } \\
\text { (\% increase) }\end{array}$ & $\begin{array}{c}\text { Search } \\
\text { step }\end{array}$ & $\begin{array}{c}\text { FS } \\
\text { bks }\end{array}$ & $\begin{array}{c}\text { MV } \\
\text { bits }\end{array}$ \\
\hline Bicycle & 131.8 & $455.2(8.5 \%)$ & 1.54 & 3.1 & 1960 \\
\hline Cheer & 119.6 & $505.7(8.9 \%)$ & 1.23 & 6.4 & 1860 \\
\hline Flower & 271.5 & $225.5(2.0 \%)$ & 0.57 & 1.4 & 951 \\
\hline Football & 177.3 & $198.3(13.6 \%)$ & 1.23 & 2.1 & 2035 \\
\hline Mobile & 356.7 & $331.8(1.7 \%)$ & 0.55 & 0.7 & 793 \\
\hline
\end{tabular}

TABLE VI

EXPERIMENTAL RESULTS OF Five TeSt Video SEQuences with Algorithm MRST

\begin{tabular}{l||c|c|c}
\multicolumn{1}{c||}{ Video } & \multicolumn{3}{c}{ Proposed algorithm MRST } \\
\cline { 2 - 4 } & $\begin{array}{c}\text { Speed- } \\
\text { up }\end{array}$ & $\begin{array}{c}\text { MSE } \\
\text { (\% increase) }\end{array}$ & $\begin{array}{c}\text { MV } \\
\text { bits }\end{array}$ \\
\hline Bicycle & 150.4 & $448.7(7.0 \%)$ & 2107 \\
\hline Cheer & 231.5 & $492.8(6.1 \%)$ & 1957 \\
\hline Flower & 287.8 & $224.6(1.6 \%)$ & 958 \\
\hline Football & 208.4 & $186.7(6.9 \%)$ & 2219 \\
\hline Mobile & 314.5 & $332.6(1.9 \%)$ & 786 \\
\hline
\end{tabular}

To gain more insights, we plot the frame-by-frame speedup factor by using the $\log$ search, S2, ST2, and MRST 


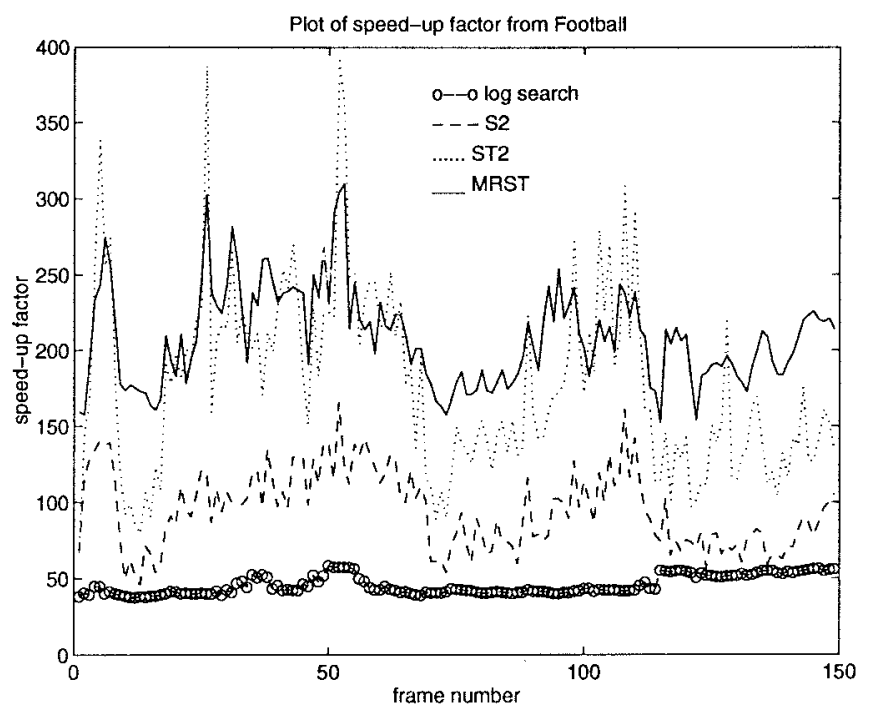

(a)

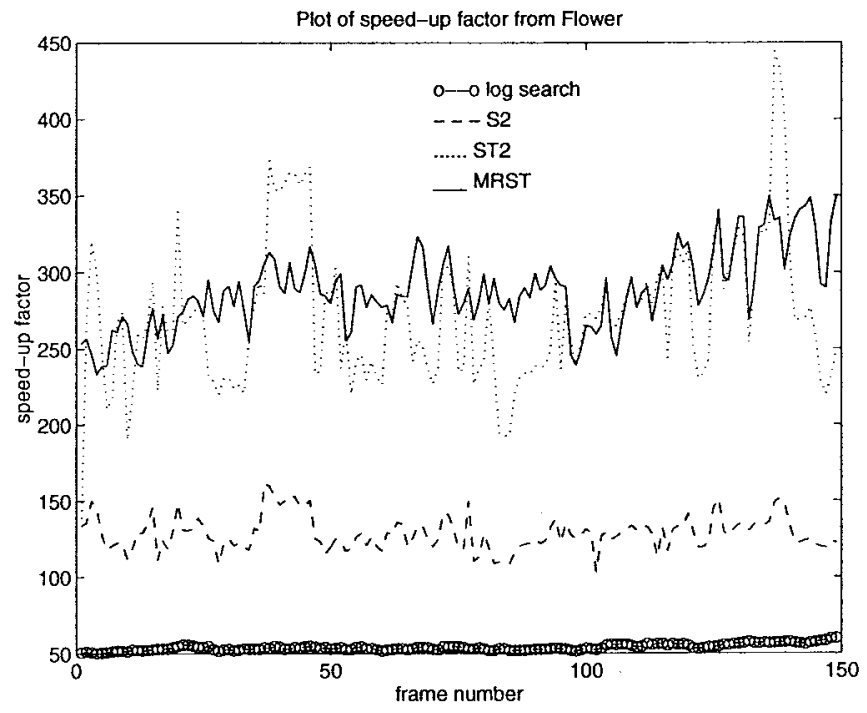

(b)

Fig. 6. The plot of the speed-up factor as a function of the frame number with log search, S2, ST2 and MRST: (a) football and (b) flower.

for "football" and "flower" sequences in Fig. 6(a) and (b), respectively. We see that MRST provides the highest speedup factor for most frames in both sequences. Besides, we observe from the plots that ST2 has a high fluctuation in the speed-up factor. This is due to the use of a fixed $T H_{1}$ for all frames, where the value of $T H_{1}$ may be too small or too big for some frames. On the other hand, MRST gives a smaller fluctuation in the plot due to the adaptive nature of $T H_{1}$ for each frame.

As a tradeoff of fast MV estimation in MRST, we observe only small MSE increase (between 2-7\%) in comparison with full search. It supports our claim that the multiresolutionspatio-temporal correlations can be effectively used to reduce the computational cost without sacrificing much in MSE. For "flower" and "mobile" sequences, the results of ST2 and MRST are similar, while for "bicycle," "cheer," and

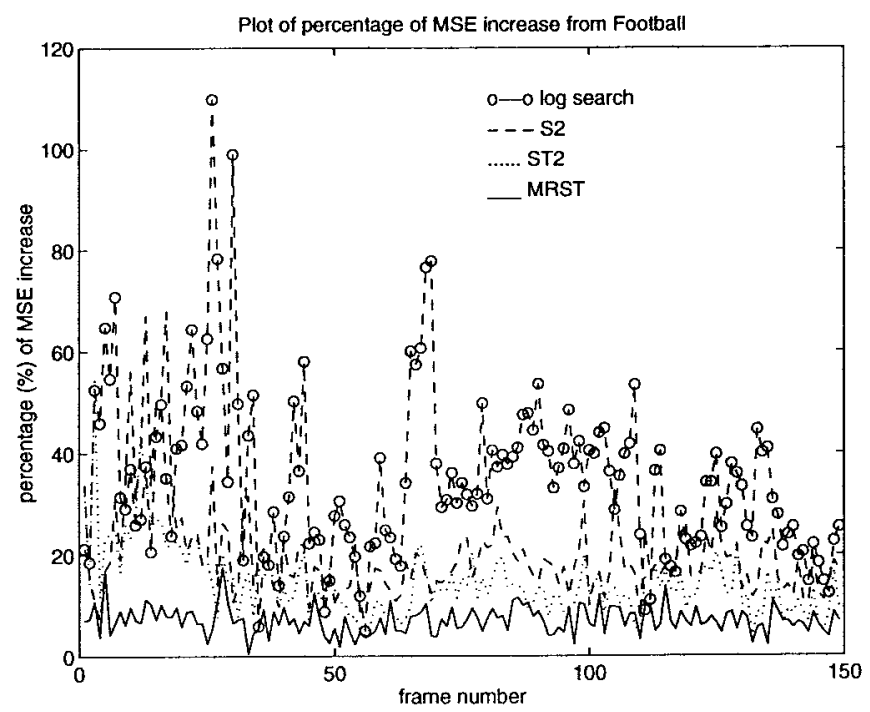

(a)

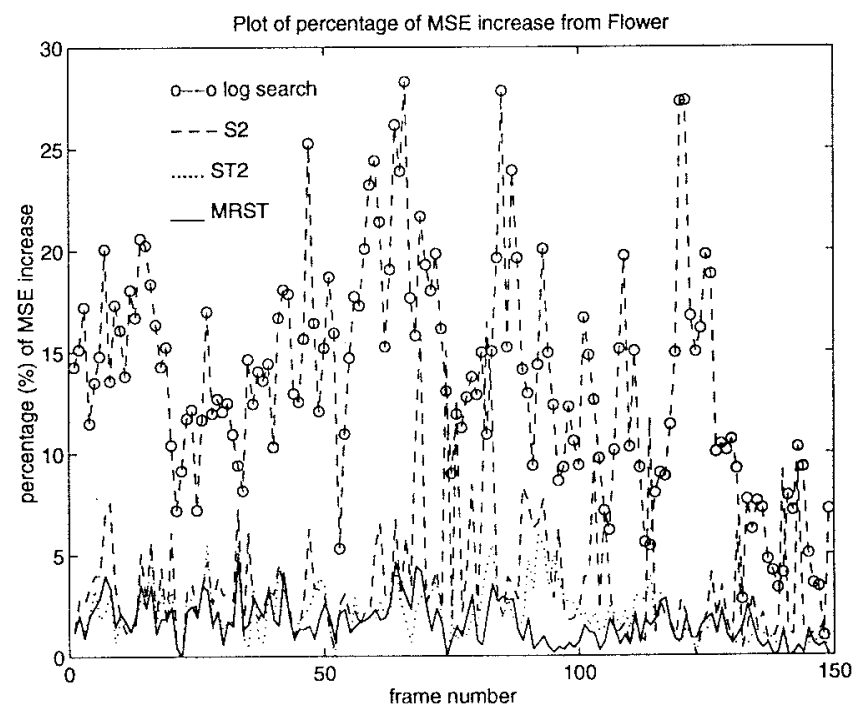

(b)

Fig. 7. The plot of percentage of MSE increase as a function of the frame number with log search, S2, ST2 and MRST: (a) football and (b) flower.

"football," MRST gives a better result in terms of a higher speed-up factor and a lower MSE value. Note also that the MSE difference between ST1 and ST2 is very small. This justifies the idea of exploiting the block subsampling technique in ST2. The frame-by-frame plots of percentage of MSE increase with the log search, S2, ST2, and MRST algorithms for "football" and "flower" are given in Fig. 7(a) and (b), respectively. It is clear that the percentages of MSE increase from MRST is much smaller than those from other algorithms. Algorithm MRST not only gives smaller MSE increases in average but also provides smaller deviations in MSE increase. This implies that the quality of the output video obtained from MRST should be better in consecutive displays.

In Tables II-V, the search step number gives us information about how close the best MV candidate is to the final MV. It 
is also clear that a smaller number of search steps implies a higher speed-up factor. Even though the maximum step number $S$ is set to ten, most blocks require only a few steps to reach the final MV. The "flower" and "mobile" sequences have the averaged step number even less than one, since there are many blocks with $M A D_{0} \leq T H_{1}$ that do not require the local search around process.

For the column of "FS bks," we see that the numbers are relatively small compared to the total number of blocks (330) used in full search. The "FS bks" number gives us a rough idea of the occurrence of motion discontinuity or regions that cannot be well represented by those in neighboring blocks or frames due to occlusion, zooming, mixed motions, etc. The "FS bks" and search step numbers are two main factors which determine the speed-up factor. For example, even though the "cheer" sequence has a search step less than that of the "bicycle" sequence, it has a larger number in "FS bks" and, therefore, a lower speed-up factor.

Next, we examine the column of MV bits. We see from tables that all five proposed algorithms use fewer bits in the coding of MV's than full search. This is a direct consequence of a smooth motion field by using the spatial correlation for MV estimation. The temporal correlation does not play an important role in MV coding since the coding is based on spatial differentials as specified by MPEG. Neither full search nor log search uses the MV information from neighboring blocks. In both methods, each block is treated independently of others. This may cause MV's to jump around in some regions and result in a higher number of MV bits. We also observe that, for "bicycle," "cheer," and "football," the MV bits of MRST are a little larger than those from ST2, which could have resulted from the use of MV's obtained from full search at the coarsest level.

Finally, we consider the coding of residual errors to obtain the rate-distortion (R-D) plot. We do not go through the whole coding process of MPEG which contains bit stream syntax, layered structure, and so on. Instead, we only perform MV differential coding and DCT residual error coding, and add the numbers of bits together to represent the "rate" with the unit of $\mathrm{b} / \mathrm{p}$ (bits per pixel). The distortion is represented by PSNR, which is computed via MSE measured from the final reconstructed images (with residual coding). Two R-D plots for "football" and "flower" sequences are demonstrated in Fig. 8(a) and (b), respectively. In both R-D plots, we see a small deterioration from the results of our algorithms in comparison with full search while the results of the log search are much worse. The results of MRST and ST2 are very similar, and they are both better than that of S2. For the "football" sequence, the R-D plot of ST2 is in fact a little better than that of MRST despite a larger MSE. The reason could be that in some regions the ST2 gives higher correlated residual errors so that fewer bits are required by DCT. For the "flower" sequence, the results of ST2 and MRST are so similar that they appear to coincide with each other. To conclude, the proposed algorithm MRST provides a very fast MV estimation procedure while maintaining a good performance in terms of MSE as well as the rate-distortion tradeoff.

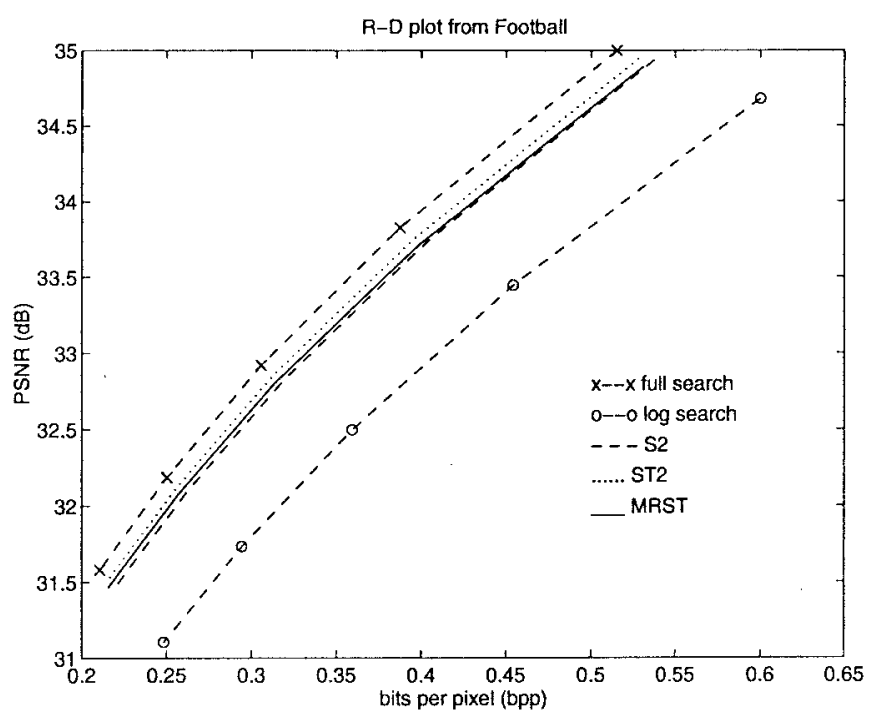

(a)

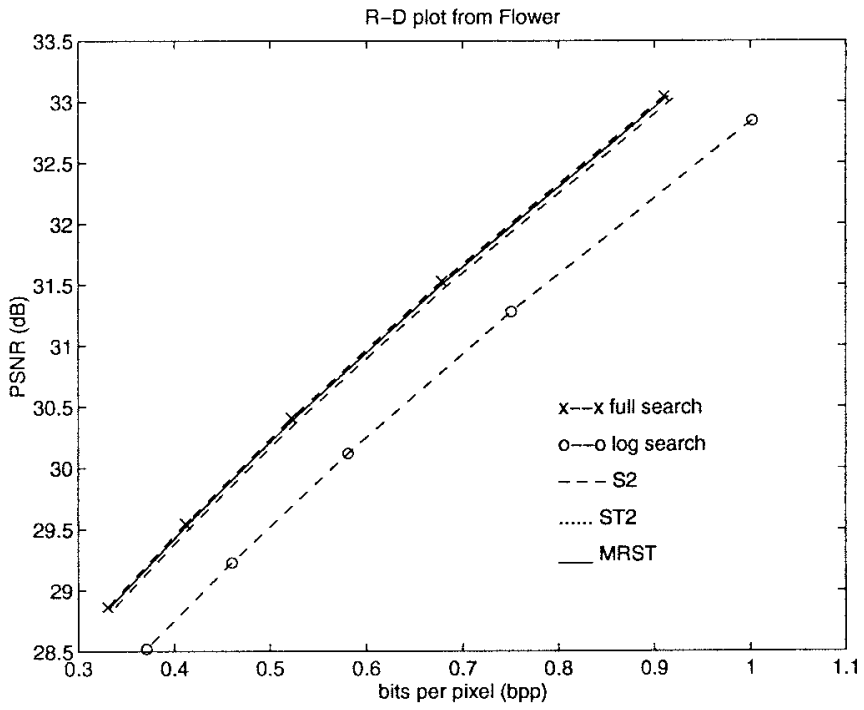

(b)

Fig. 8. Comparison of the rate-distortion performance with full search, log search, S2, ST2, and MRST, where the rate is represented by bits per pixel (bpp) while distortion is represented by PSNR (dB): (a) football and (b) flower.

\section{CONCLUSIONS}

bIn this work, we first introduced two fast MV estimation algorithms S1 and S2 based on spatial correlation of MV's between adjacent blocks and then incorporated the temporal information to obtain Algorithms ST1 and ST2. We finally proposed the ultimate algorithm MRST by combining the multiresolution scheme with spatio-temporal correlations. The initial MV candidates of MRST are selected from the corresponding coarser-level block as well as spatially and temporally neighboring blocks at the same level. We showed with experimental results that the proposed algorithm MRST has a speed-up factor ranging from 150 to 310 with only $2-7 \%$ MSE increase and a similar ratedistortion performance in comparison with full search. Therefore, this algorithm can be very useful for real-time video encoding applications. Theoretical analysis of the 
proposed algorithms may be performed based on a certain model such as the AR model to characterize correlations of the motion field, which is an interesting topic for future research.

\section{REFERENCES}

[1] M. Bierling, "Displacement estimation by hierarchical block matching," in Proc. SPIE Visual Communications and Image Processing '88, vol. 1001, pp. 942-951.

[2] F. Dufaux and M. Kunt, "Multigrid block matching motion estimation with an adaptive local mesh refinement," in Proc. SPIE Visual Commun. and Image Processing '92, vol. 1818, pp. 97-109.

[3] D. LeGall, "MPEG: a video compression standard for multimedia applications," Commun. ACM, vol. 34, pp. 46-58, Apr. 1991.

[4] M. Ghanbari, "The cross-search algorithm for motion estimation," IEEE Trans. Commun., vol. 38, pp. 950-953, July 1990.

[5] J. R. Jain and A. K. Jain, "Displacement measurement and its application in interframe image coding," IEEE Trans. Commun., vol. COM-29, pp. 1799-1808, Dec. 1981

[6] T. Koga, K. Iinuma, A. Hirano, Y. Iijima, and T. Ishiguro, "Motion compensated interframe coding for video conferencing," in Proc. Nat. Telecommunication Conf., pp. G5.3.1-5.3.5, Nov. 29-Dec. 3, 1981.

[7] J. Li, X. Lin, and Y. Wu, "Multiresolution tree architecture with its application in video sequence coding: A new result," in Proc. SPIE Visual Communications and Image Processing '93, vol. 2094, pp. 730-741.

[8] R. Li, B. Zeng, and M. L. Liou, "A new three-step search algorithm for block motion estimation," IEEE Trans. Circuits Syst. Video Technol., vol. 4, pp. 438-442, Aug. 1994.

[9] B. Liu and A. Zaccarin, "New fast algorithms for the estimation of block motion vectors," IEEE Trans. Circuits Syst. Video Technol., vol. 3, pp. 148-157, Apr. 1993.

[10] MPEG1, "Coding of moving pictures and associated audio," Tech. Rep., Committee Draft of Standard ISO 11172, 1990.

[11] MPEG2, "Information technology-generic coding of moving pictures and associated audio," Tech. Rep., ISO/IEC 13818-2, Committee Draft, Mar. 1994.

[12] H. G. Musmann, P. Pirsch, and H.-J. Grallert, "Advances in picture coding," Proc. IEEE, vol. 73, pp. 523-548, Apr. 1985.

[13] M. T. Orchard, "A comparison of techniques for estimating block motion in image sequence coding," in Proc. SPIE Visual Communications and Image Processing '89, vol. 1199, pp. 248-258.

[14] A. Puri, H.-M. Hang, and D. L. Schilling, "An efficient block matching algorithm for motion-compensated coding," in Proc. IEEE ICASSP '87, pp. 25.4.1-25.4.4.

[15] R. Srinivasan and K. R. Rao, "Predictive coding based on efficient motion estimation," IEEE Trans. Commun., vol. COM-33, pp. 888-896, Aug. 1985.

[16] K. M. Uz, M. Vetterli, and D. LeGall, "Interpolative multiresolution coding of advanced television with compatible subchannels," IEEE Trans. Circuits Syst. Video Technol., vol. 1, pp. 86-99, Mar. 1991.

[17] K. Xie, L. V. Eycken, and A. Oosterlinck, "A new block-based motion estimation algorithm," Signal Processing: Image Commun., vol. 4, pp. 507-517, 1992.
[18] S. Zafar, Y.-Q. Zhang, and J. S. Baras, "Predictive block-matching motion estimation for TV coding-Part I: Inter-block prediction," IEEE Trans. Broadcast., vol. 37, pp. 97-101, Sept. 1991.

[19] S. Zafar, Y. Q. Zhang, and B. Jabbari, "Multiscale video representation using multiresolution motion compensation and wavelet decomposition," IEEE J. Select. Areas Commun., vol. 11, pp. 24-35, Jan. 1993.

[20] Y.-Q. Zhang and S. Zafar, "Predictive block-matching motion estimation for TV coding - Part II: Inter-frame prediction," IEEE Trans. Broadcast., vol. 37, pp. 102-105, Sept. 1991.

[21] _ "Motion-compensated wavelet transform coding for color video compression," IEEE Trans. Circuits Syst. Video Technol., vol. 2, pp. 285-296, Sept. 1992.

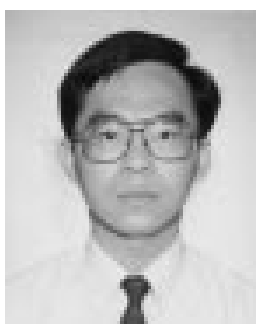

Junavit Chalidabhongse (S'91-M'96) received the B.E. degree from the Chulalongkorn University, Bangkok, Thailand, in 1988 and the M.S. and Ph.D degrees from the University of Southern California, Los Angeles, in 1989 and 1996, respectively, all in electrical engineering.

$\mathrm{He}$ is currently working as a Research Associate in the Department of Electrical Engineering at the University of Southern California, Los Angeles. His research interests include image and video compression, low bit-rate video coding, visual communication, and image processing.

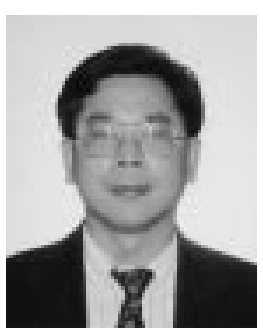

C.-C. Jay Kuo (S'83-M'86-SM'92) received the B.S. degree from the National Taiwan University, Taipei, in 1980 and the M.S. and Ph.D. degrees from the Massachusetts Institute of Technology, Cambridge, in 1985 and 1987, respectively, all in electrical engineering.

From October 1987 to December 1988, he was Computational and Applied Mathematics (CAM) Research Assistant Professor in the Department of Mathematics at the University of California, Los Angeles. Since January 1989, he has been with the Department of Electrical Engineering-Systems and the Signal and Image Processing Institute at the University of Southern California, where he currently has a joint appointment as Associate Professor of Electrical Engineering and Mathematics. His research interests are in the areas of digital signal and image processing, video coding, wavelet theory and applications, multimedia technologies, and large-scale scientific computing. He has authored more than 200 articles.

Dr. Kuo is a member of Sigma Xi, SIAM, ACM, and a Fellow of SPIE. He serves as Associate Editor for IEEE TRAnsactions on IMAge Processing and IEEE TRansactions on Circuits and Systems for Video TeChNOLOGy and is on the editorial board of the Journal of Visual Communication and Image Representation. He received the National Science Foundation Young Investigator Award (NYI) and Presidential Faculty Fellow (PFF) Award in 1992 and 1993, respectively. 